\title{
PERFORMANCE OF TWO BRANCH SPACE AND POLARIZATION DIVERSITY AT 900 MHZ. ${ }^{1}$
}

\author{
Silvia Ruiz-Boqué, Marc.Vilades, J.Rodriguez \\ Dep. Teoria del Senyal i Comunicacions, ETSETB, Barcelona, Spain \\ E-mail: silvia@xaloc.upc.es
}

\begin{abstract}
This work summarizes the results of a measurement campaign undertaken to evaluate the performance of two branch space and polarization diversity schemes at $900 \mathrm{MHz}$ in different environments (urban, suburban, rural flat and rural hilly). A total number of 783 runs were made covering these sites and using different transmitter antenna (vertical polarized roof-top antenna, hanheld randomly inclined) at differents speeds (walking, by car at 30 up to $80 \mathrm{~km} / \mathrm{h}$ ). Comparison between the two types of diversity schemes have been made through calculation of the statistics of mean received signal level, diversity gain, incremental diversity gain and envelope cross-correlation. From these parameters many conclusions can be extracted which will be given in this paper.
\end{abstract}

\section{INTRODUCTION}

Multipath propagation and shadowing objects between base station and mobile are the responsible of signal fading in mobile communications. The required fading margin when designing the communications system, is approx.15 $\mathrm{dB}$ if no diversity is applied. Several diversity techniques can be used at the base station or the mobile unit to reduce the required fading margin. Taking into account that usually the maximum power transmitted by the mobile unit is lower than the maximum power of the base station (differences around 8-10 dB between up-link and down-link) it is most advantegous to reduce the multipath fading margin on uplink by the use of base-station antenna diversity.

Up to this moment the diversity technique used more frequently in Spain for GSM system is space diversity with antenna separations around $20 \lambda\left(90^{\circ}\right.$ broadside $)$ and $80 \lambda\left(0^{\circ}\right.$ endfire) for horizontal spacing. This requires a big tower to support the antennas increasing installation derived costs and it is not visually attractive.

A way to obtain two independently fading signals without the need for physical separation between antennas is by the use of polarization diversity (with a single compact antenna capable of receiving dual polarization). But there are few studies reported in literature about polarization diversity. This is due to the fact that cross-polarization discrimination
(XPD) reduces diversity gain and also the envelope correlation coefficient is usually higher than in space diversity. By the use of hand-helds, the antenna will be inclined from the vertical and then the transmitted power is partitioned between vertical and horizontal polarizations and small XPD is expected. This benefits polarization diversity and degrades the performance of space diversity.

Then it seems interesting to test and compare the performance of both, space and polarization diversity (SD and PD) techniques, as has been done in this work in a wide variety of environments, and considering equal gain combining technique.

\section{MEASURED SITES}

Along this study four different sites were measured:

- urban site (Barcelona) : 127 runs

- suburban site (Cerdanyola del Valles) : 199 runs

- rural hilly terrain (Mollet del Valles) : 170 runs

- rural flat terrain (Zaragoza) : 287 runs

at different speeds and using different transmitter antennas (roof antenna or hanheld antenna). At each site as many runs as possible were done considering different situations: LOS/NLOS, close-medium-far distance from base station, in the middle or in the limit of the coverage area, paralelperpendicular to the base stations antennas, etc. Each run covers a distance of around $250 \mathrm{~m}$.

\section{CAlCulated PARAMETERS}

The efficency of the diversity scheme relies on the detection of two independent fading signals with comparable mean signal levels.

a) The cross-correlation between envelopes of the signals received at the antennas is often used to measure the independence. A cross correlation coefficient of 0.7 or lower is accepted [1]. It has been calculated for each run (for SD and $\mathrm{PD}$ ) removing the variation of the local mean. For so

${ }^{1}$ this work has been supported by Airtel company (Spain) 
doing a moving average technique is employed with a sliding window of $20 \lambda$. The cross correlation between the fast fading components of the signals is calculated using the relationship:

$$
\rho=\frac{\sum_{i=1}^{N}\left[r_{1}(t)-\bar{r}_{1}(t)\right]\left[r_{2}(t)-\bar{r}_{2}(t)\right]}{\sqrt{\sum_{i=1}^{N}\left[r_{1}(t)-\bar{r}_{1}(t)\right]^{2}} \sqrt{\sum_{i=1}^{N}\left[r_{2}(t)-\bar{r}_{2}(t)\right]^{2}}}
$$

where $r_{1}(t)$ and $r_{2}(t)$ are the instantaneous levels of the normalized fast fading signals for the two branches and $r_{1}(t)$ and $r_{2}(t)$ are their respective means.

Also for each site the statistics of the crosscorrelation coefficient for SD and PD is calculated. This summarizes the behaviour in the site.

b) Statistics of combined signal through the calculation of the following parameters:

- mean and median of received combined power with an equal gain combining technique. For both diversity types the overall mean signal level is calculated for each run by averaging the power of the recorded data (without removing local mean).

- the CDF of the local area means and of the difference between local area means of the combined received power is also calculated averaging the combined signal each $20 \lambda$.

This gives information about the ability of each diversity technique to supress deep fades.

c) The diversity gain defined as the difference in signal level between one branch and the received at the output of the diversity combiner for a given error probability. This gives an indication of the reduction in fading depth and therefore the required fading margin in designing the communication system. Also the PDF of the diversity gain is calculated for each site to compare environments. The way to calculate the diversity gain from signal strength samples is the following:

- received power considering isotropic antenna is calculated (considering for each of the four branches the antenna gain,cables/connectors losses, preamplifier gain, spectrum analyser calibration function and $\mathrm{A} / \mathrm{D}$ conversion).

- the file is subdivided in blocks of 20 wavelength $(6,57 \mathrm{~m})$ and for each block the diversity gain is calculated.

- the error rate is defined as:

$$
p_{b}=0.5 \operatorname{erf}\left(\sqrt{\frac{P}{N}}\right)
$$

where $\mathrm{P}$ can be the received power from branch $1\left(P_{1}\right)$, from branch $2\left(P_{2}\right)$ or the sum in linear of the received power in the two branches (Psum $\left.=P_{1}+P_{2}\right) . N$ is an arbitrary constant adjusted to give sufficient statistics in the interesting region of $\mathrm{pb}$ (assuming that SNR in the measured samples is sufficiently high that the noise can be neglected and adjusted to arbitrary level in the eq. for $\mathrm{pb}$ ).
- a mean error of $2 \%$ is considered and then the following expression has to be acomplished:

$$
<p_{b}>=\frac{1}{M} \sum_{i=1}^{M} 0.5 \operatorname{erfc}\left(\sqrt{\frac{P_{\text {sum }, i}}{N}}\right)=0.02
$$

being $M$ the number of points contained in $20 \lambda$

Maintaining the value of $\mathrm{N}$ and taking the signal from one antenna, the increment/decrement of power level is calculated to produce the same mean error rate.

$$
<p_{b}>=\frac{1}{M} \sum_{i=1}^{M} 0.5 \operatorname{erfc}\left(\sqrt{\frac{P_{1, i} G}{N}}\right)=0.02
$$

- this adjustement $\mathrm{G}(\mathrm{dB})$ represents the diversity gain $(\mathrm{G}>0$ means that the diversity is effective in suppressing fast fading compared to the use of a single antenna).

- The process is repeated for all the blocks in the run.

d) The incremental diversity gain from PD with respect SD is calculated for each run and considering blocks of $20 \lambda$. The way to obtain the incremental diversity gain is similar to the method for obtaining the diversity gain but using as reference the combined signal for space diversity instead of the signal from one single antenna. The incremental diversity gain $\Delta \mathrm{G}$ (in $\mathrm{dB}$ ) represents the local improvement or degradation in performance between polarisation and space diversity (a positive value means that polarization diversity is more effective in suppresing fast fading than space diversity).

e) Statistics of the difference between the local means of the two space diversity branches have also been calculated.

\section{MEASUREMENT TECHNIQUE.}

To give an accurate comparison between techniques, the signals from the 4 branches were measured simultaneously by the use of 4 receivers and at a rate capable to tracke Rayleigh fading at $900 \mathrm{MHz}$ and at $80 \mathrm{~km} / \mathrm{h}$. The receivers consist of a GSM bandpass filter followed by an amplifier and an HP spectrum analyser. The output of the four analysers were input to a DAT recorder which can record four channels simultaneously at the required sampling rate. Specific software to detect and store received signals was designed.

\section{OBTAINED RESULTS}

\section{A. Envelope Cross-Correlation coefficient $\rho$}

In general the envelope cross-correlation coefficient is lower in space than in polarization diversity branches.

For SD technique the lowest values were obtained in the urban area where there are many scatterers in the vecinity of 
both mobile antenna and base station. In second place we have the suburban area and then rural hilly and rural flat. In rural flat area most of the measures were in LOS with no scatteres (no buildings and flat terrain) and for this reason the signal is more correlated.

For PD technique again the lowest values were obtained in urban area [2] being the values for the other three areas similar. Also for this technique in general higher values were obtained when using the roof antenna (sometimes higher than 0.7) compared to the hanheld antenna and therefore there seems to be a correlation between $\rho$ and the antenna inclination.

a) urban site : In this environment the correlation coefficient is in general low for both diversity techniques (all runs with $\rho<0.7$ ). It is observed that in the case of PD the values are higher when using roof-top antenna compared to handheld antenna.

b) Suburban site: All the runs are below 0.7 for $\mathrm{SD}$ while for $\mathrm{PD}$ the $20 \%$ of the runs are above this value.

This means that the separation between antennas for the SD technique is good enough to provide sufficient decorrelation between received signals. In the case of PD technique: using hanheld the correlation coeficient was always lower than 0.7 but when using roof-top antenna the $38 \%$ of the runs show a coefficient higher than 0.7 .

c) Rurall hilly site: $90 \%$ of the runs are below 0.7 for SD while for $\mathrm{PD}$ the $32 \%$ of the runs are above this value.

For PD the correlation coefficient was always lower than 0.7 when using hanheld while the $47 \%$ of runs with roof-top antenna show a coefficient higher than 0.7 .

d) rural flat site: This is the site where higher values of the correlation coefficient were obtained due to the fact that most of the measures were in LOS (flat area with no buildings). $83 \%$ of the runs are below 0.7 for SD while for PD the $30 \%$ of the runs are above this value. For PD the correlation coefficient is always lower than 0.7 when hanheld while using roof-top antenna the $54 \%$ of runs show a value higher than 0.7 .

\section{B. Statistics of combined signals}

In most of the measures the mean received power is higher for SD than for PD system, except for rural flat area. The difference depends on the type of antenna used due to the angle of inclination of the transmitter antenna. In general when using roof antenna, the signal is transmitted in vertical polarization, this is the same polarization as the antennas used for SD, while a loss in received signal is obtained for the $\pm 45^{\circ}$ antennas used for PD. As the transmitter antenna is tilted (with the hanheld), a reduction in received power is observed at the $\mathrm{SD}$ and then the difference between mean received powers decreases.
From the point of view of PD the worst results were obtained for suburban site, urban and rural hilly sites are comparable being the best environment rural flat area. Nevertheless de difference in mean received power are quite low meaning that both techniques can be considered comparable from the point of view of mean received power.

a) suburban: For mean received power $<-75 \mathrm{dBm}$ ( $80 \%$ of the runs) there is a difference of around $4 \mathrm{~dB}$ in mean received power (SD higher). This difference is reduced being both levels comparable for mean received power between -40 and $-70 \mathrm{dBm}$.

\begin{tabular}{|c|c|c|c|c|}
\hline$(1)$ (1) & 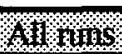 & 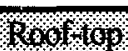 & The ed ear & Haldowalk \\
\hline $00 \%$ & 7 & 8 & 4.5 & 5.5 \\
\hline $50 \%$ & 4 & 5.5 & 3 & 4 \\
\hline $60 \%$ & 0 & 0.5 & 0 & -1 \\
\hline
\end{tabular}

Differences bettween mean power are high when using roof-top antenna and decrease when using the hanheld. Considering the same run but using different antennas: There is a reduction around $3 \mathrm{~dB}(6 \mathrm{~dB})$ in mean received power for $\mathrm{PD}$ (SD) when a run is done walking with the hanheld compared to the run by the car and roof antenna. There is a reduction around 7-9 $\mathrm{dB}(9-11 \mathrm{~dB})$ for PD (SD) when a run is done by car but with the handheld compared to the run by car and roof antenna.

b) rural hilly: The differences bettween mean received power are high when using roof-top antenna and decrease when using the hanheld (for both inside vehicle or walking runs). With the hanheld inside the car for the $30 \%$ of measures the signal is higher for polarization diversity than for space diversity.

\begin{tabular}{|c|c|c|c|c|}
\hline Cep & All nuns & Roefsop & Hilloldear & 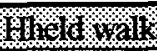 \\
\hline 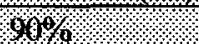 & 5 & 5.5 & 4 & 4.5 \\
\hline 50\% $\%$ & 2.5 & 3 & 1 & 3.5 \\
\hline$\frac{16 \%}{6 \%}$ & -1 & $\overline{1}$ & -3 & 2.5 \\
\hline
\end{tabular}

c) urban: The difference bettween mean received power are slightly higher when using roof-top antenna but in all the cases the differences are between $2-3 \mathrm{~dB}$ and can be concluded that no clear influence on the type of antenna used is detected.

\begin{tabular}{|c|c|c|c|c|}
\hline CBC diff(dB) & 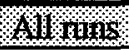 & Roortioge & 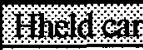 & 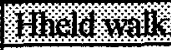 \\
\hline $90 \% \%$ & 4.5 & 5 & 3.5 & 3.5 \\
\hline $50 \%$ & 2 & 3 & 1.5 & 2 \\
\hline $10 \%$ & -1 & 0.5 & -1.5 & 0 \\
\hline
\end{tabular}

d) rural flat: Mean combined received power level is in general higher for $\mathrm{PD}$ than for SD.

\begin{tabular}{|c|c|c|c|c|}
\hline Crof dín $(A B)$ & 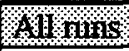 & $\mathrm{RoB} / \mathrm{HOg}$ & Priefdear & ofundu \\
\hline $60 \% \%$ & 1.5 & 2 & 0.5 & 5 \\
\hline $80 \%:$ & -1 & 0 & -2 & 0 \\
\hline $10 \%$ & -4 & -2 & -6.5 & -4 \\
\hline
\end{tabular}


When using the hanheld the $80 \%$ of runs show a mean received power higher for polarization diversity than for space diversity.

\section{Diversity gain ( $G$ in $d B)$}

For SD the diversity gain is calculated as the reduction in received power level necessary to maintain the error probability to a prefixed value when two antennas are considered compared to the case of using only one. For PD is calculated in the same way but comparing the combined signal (from the two antennas used for PD) with the signal received at one branch of the SD system (antenna with vertical polarization).

In the case of SD the CDF of the diversity gain is the same for the four sites, and $G$ experiments a low variation when the antenna is tilted. Then, when the hanheld is used there is a significant reduction in mean combined received power (as we have seen previously) that does not afect to $G$, being the system able to reduce deep fades.

For PD the CDF of diversity gain changes considerable from one site to another. The worst case is suburban site, then rural hilly site followed by urban site and the best is rural flat site. Also $\mathrm{G}$ experiments a considerable increase when the antenna is tilted (with hanheld an increase of around $4 \mathrm{~dB}$ is obtained).

In conclusion, differences between PD and SD techniques are significative only in the suburban environment with roof top antenna. In the rest of environments both tehcniques show similar gains, being PD better when using handheld.

a) suburban site: In this case $G$ was always higher for SD than $\mathrm{PD}$. The difference is around $4-5 \mathrm{~dB}$ when roof-top antenna is considered and it is reduced to around $2 \mathrm{~dB}$ for hanheld antenna. With roof antenna, $50 \%$ of measures show a negative value for PD (no advantage is obtained when using diversity). With hanheld only the $5 \%$ of runs show negative diversity gain.

\begin{tabular}{|c|c|c|}
\hline 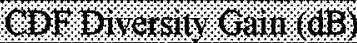 & 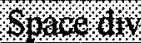 & 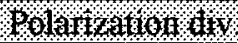 \\
\hline 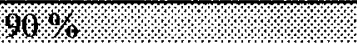 & $8 \mathrm{~dB}$ & $6 \mathrm{~dB}$ \\
\hline $50 \%$ & 5 & 2 \\
\hline $10 \%$ & 3 & -3 \\
\hline
\end{tabular}

b) rural flat site: There is a $20 \%$ of measures in which $\mathrm{G}$ is higher for PD than for SD, and in general the difference in G between both diversity techniques is lower than in the suburban site. With roof top antenna $G$ is always higher for $\mathrm{SD}$ (around $2 \mathrm{~dB}$ of difference) and $20 \%$ of runs show $\mathrm{G}>0$ $\mathrm{dB}$ with $\mathrm{PD}$ technique. With hanheld only in the $25 \%$ of runs the gain for SD was higher than for PD and only the $5 \%$ of runs show $G<0 d B$. The values for $G$ were very similar in this case being PD around $1 \mathrm{~dB}$ higher than SD.

\begin{tabular}{|c|c|c|}
\hline (1) & Space $/ 11$ & 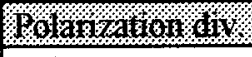 \\
\hline 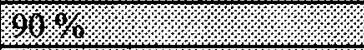 & $7 \mathrm{~dB}$ & $8 \mathrm{~dB}$ \\
\hline $50 \%$ & 5 & 3.8 \\
\hline $10 \%$ & 2 & -1 \\
\hline
\end{tabular}

c) urban site: There is a $15 \%$ of measures in which $G$ is higher for PD than for SD. With roof top antenna the gain for $\mathrm{PD}$ is around $2 \mathrm{~dB}$ lower than the gain for $\mathrm{SD}$. This difference is reduced when handheld is considered. The differences in $\mathrm{G}$ are not significant between both techniques.

\begin{tabular}{|c|c|c|}
\hline CDP Drrersily Garin (dB) & 51000 & Polarizationuctuy \\
\hline 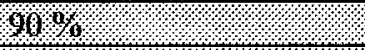 & $8 \mathrm{~dB}$ & $8 \mathrm{~dB}$ \\
\hline $50 \% \%$ & 6 & 5 \\
\hline $10 \% \%$ & 4 & 2 \\
\hline
\end{tabular}

d) rural hilly site: In most of the measures $G$ is higher for PD than for SD (even with roof top antenna). This difference increases considerably when hanheld antenna is used.

\begin{tabular}{|c|c|c|}
\hline 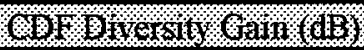 & Specedily & 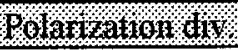 \\
\hline 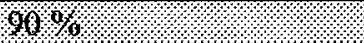 & $8 \mathrm{~dB}$ & $12 \mathrm{~dB}$ \\
\hline $50 \%$ & 5 & 6 \\
\hline $10 \%$ & 3 & 3 \\
\hline
\end{tabular}

\section{$D$ Incremental diversity gain ( $\Delta G$ in $d B$ ).}

From the point of view of $\Delta G$ the worst environment is the suburban area (the same conclusion looking at the parameters previously commented). Urban and rural hilly areas show similar behaviour being slightly better the urban area, specially when roof antenna is used.

a) suburban site: In general $\Delta G<0 \mathrm{~dB}$. This means that to have the same mean error a reduction in power level can be considered when using SD.

b) nural hilly site: In this area it is possible to say that both techniques are comparable and that PD is better than SD if handheld is used.

c) urban site: In this area the absolute values of $\Delta G$ show that both diversity techniques are comparable and when hanheld is used PD is in general a bit better.

d) rural flat site In this area $\Delta G$ is usually positive.

\section{Difference between branches}

The CDF of the difference between the local means of the two SD branches have been calculated in order to know if they are equivalent or not when calculating $\mathrm{PD}$ gain Naming SD antennas by $A 1$ and $A 2$, the statistics for the difference have been calculated as A2 - A1 level (each 20 $\lambda$ ). When PD gain was calculated we used $A 1$ as reference. 
It is expected that no significative differences will appear between the received power levels at both antennas. This is what happens for urban, suburban an rural hilly terrain. In the case of rural flat terrain most of the measures were done in the two major roads close to the base station where one antenna was at closer distance from the car than the other. So in this case the level of A2 was in general higher than the level in A1. Then the antenna with lower level was the reference when calculating PD gain.

a) suburban site: Signal levels at both antennas are similar being $\mathrm{A} 1$ slightly higher than $\mathrm{A} 2$.

\begin{tabular}{|c|c|c|c|c|}
\hline (EBf d d I & All rains & 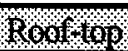 & III & 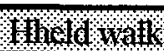 \\
\hline $90 \%$ \% & 2 & 2.5 & 2 & 3 \\
\hline $50 \% \%$ & -0.4 & -0.5 & -0.5 & 0 \\
\hline $10 \%$ & -2.8 & -3 & -2.4 & -2 \\
\hline
\end{tabular}

b) rural hilly site: Levels at both antennas are similar.

\begin{tabular}{|c|c|c|c|c|}
\hline 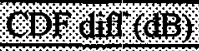 & All rams & Roortore & Ifleld car & Mhero walk \\
\hline $90 \%$ - & 2.5 & 2.5 & 2 & 2.5 \\
\hline $61 \% \%$ & 0.2 & 0.2 & 0 & 1.8 \\
\hline $10 \%$ & -2 & -2 & -2.5 & 1 \\
\hline
\end{tabular}

c) urban site: Signal levels at both antennas are similar being A2 slightly higher than $\mathrm{A} 1$.

\begin{tabular}{|c|c|c|c|c|}
\hline 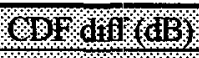 & All nums & Rooflop & Ithela car & Hineld waike \\
\hline $60 \% \%$ & 3.5 & 4 & 3 & 2.5 \\
\hline $80 \%$ & 0.5 & 0.5 & 0.2 & 0 \\
\hline $16 \%$ & -2.5 & -2.5 & -2.5 & -2.5 \\
\hline
\end{tabular}

d) rural flat site: Levels at $\mathrm{A} 2$ higher than at $\mathrm{A} 1$ were obtained. This means that lower values of $G$ would be obtained if we had used $\mathrm{A} 2$ as reference.

\begin{tabular}{|c|c|c|c|c|}
\hline 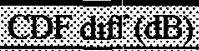 & All rans & Kooflofop & Iticlo car & Wheld watk \\
\hline $90 \%=-1.9$. & 3.5 & 3.5 & 3.2 & 3 \\
\hline $50 \%$ & 1.5 & 1 & 1.5 & 1.4 \\
\hline $18 \%$ & -1.8 & -2.5 & -1.5 & -1.5 \\
\hline
\end{tabular}

\section{CONCLUSIONS}

The results of a measurement campaign undertaken to evaluate the performance of two branch space and polarization diversity schemes at GSM band frequencies in urban, suburban, nural flat and rural hilly sites have been reported. From the parameters above studied many conclusions can be extracted.

The separation between the antennas used for SD is sufficient to provide uncorrelated signal branches. In general the envelope cross-correlation coefficient is lower in space than in polarization diversity branches. The lowest correlation coefficients were obtained in the urban area.

In most of the measures the mean received power is higher for SD than for PD. Nevertheless this difference is quite low meaning that both techniques can be considered comparable from the point of view of mean received power.

SD diversity gain is similar for the four sites tested and experiments a low variation whan the antenna is tilted. Then, for $\mathrm{SD}$, when the hanheld is used there is a significant reduction in mean combined received power that does not afect to the diversity gain, being the system able to reduce deep fades (mean diversity gains between 5 and $6.5 \mathrm{~dB}$ depending on environment). Considering PD the diversity gain changes considerable from one site to another. The worst case is suburban site, then rural hilly site followed by urban site and finally rural flat site. In this case the diversity gain experiments a considerable increase when the antenna is tilted (tipical values aroun 1-2 $\mathrm{dB}$ with rooftop antenna and aroun 3.5-6 dB with hanheld). This explains the increase in incremental diversity gain and the fact that for some environments a positive value is obtained indicating that PD is better than SD.

In urban, suburban an nural hilly terrain no significative differences appear between the received power level at the two SD branches. In the case of rural flat terrain there is a mean difference of around $1.5 \mathrm{~dB}$ between signals received at both antennas being the antenna with lower signal the one that has been used to calculate the PD diversity gain.

The results lead to the conclusion that polarization diversity will perform almost as well as or better than horizontal separation space diversity schemes in situations where random orientation of the transmitting antenna is likely (for example the use of hanhelds).

\section{References}

[1] A.Turkmani et al. "An experimental evaluation of the performance of two branch space and polarization diversity schemes at $1800 \mathrm{MHz}$ "IEEE Trans.VT, vol.44. no. 2, may 1995.

[2] R.Vaughan "Polarization Diversity in Mobile Communications" IEEE Trans.Veh.Techn.Vol.39,no.3, august 1990. 